\title{
PROPAGATION OF CYLINDRICAL SHOCK WAVES IN A NON-UNIFORM, ROTATING STELLAR ATMOSPHERE UNDER THE ACTION OF MONOCHROMATIC RADIATION AND GRAVITATION
}

\author{
Shilpa Shinde \\ Department of Applied Mathematics and Computational Sciences \\ Shri G. S. Institute of Technology and Science \\ 23, Park Road, Indore-452003, India \\ shilpa_shinde1@rediffmail.com
}

\begin{abstract}
A self-similar, theoretical model of propagation of cylindrical shock wave in a magneto- gas dynamic rotating non-uniform atmosphere in the presence of monochromatic radiation and gravitation, is considered. The result discussed depends upon the variations of the flow variables behind the shock, which are displayed graphically. A special case is considered in presence and absence of gravitation along with rotation to observe the influence of gravitation.
\end{abstract}

Keywords- Magneto-gas dynamic shock wave, Self similar motion, Monochromatic radiation.

\section{INTRODUCTION}

Recently there has been considerable interest in the study of processes occurring under the action of radiation on gaseous substances in the stellar interiors. Sedov[3] made it possible to analyze certain classes of self-similar solutions in a number of problems with disturbed energy release with the help of methods of theory of dimensionality. Onkar Nath[1] presented a theoretical model of cylindrical magneto-hydrodynamic shock waves under the action of monochromatic radiation in non-uniform stellar atmosphere, following the work of Khudyakov on the self-similar problem of motion of a gas under the action of monochromatic radiation. Nath [2] further studied a model of cylindrical shock waves in a non-uniform rotating atmosphere under the action of monochromatic radiation, where he omitted magnetic field effect. In the present paper, we have studied the propagation of cylindrical shock wave in a magneto-gas dynamic rotating non-uniform atmosphere in the presence of monochromatic radiation and gravitation. Gas is assumed to be gray and opaque and shock to be transparent. The shock is assumed to be propagating in a conducting medium at rest with density varying as $r^{\beta} \quad(-2<\beta \leq 0)$. The magnetic field distribution varies as $r^{\alpha} \quad(\alpha<0)$ and is directed tangential to the advancing shock front. The radiation flux moves through the gas with a constant intensity in the direction opposite to that of the propagation of shock wave. Further, the rotating gas does not radiate itself and energy in absorbed only behind the shock wave. Also, it is assumed that the radiation pressure and radiation energy are very small in comparison to the material pressure and energy, hence neglected. The results discussed depend upon the variations of the flow variables behind the shock, which are displayed graphically. A special study is undertaken to consider the influence of gravitation on the variations of flow parameters in the presence and absence of rotation. 


\section{EQUATIONS OF MOTION AND BOUNDARY CONDITIONS}

In accordance with the above assumptions the motion of an inviscid perfect gas in a magneto-gas dynamic rotating non uniform medium in presence of monochromatic radiation and gravitation can be described by the following system of differential equations:

$$
\begin{aligned}
& \frac{\partial \rho}{\partial t}+\frac{\partial(\rho u)}{\partial r}+\frac{\rho u}{r}=0 \\
& \frac{\partial u}{\partial t}+u \frac{\partial u}{\partial r}+\frac{1}{\rho} \frac{\partial p}{\partial r}+\frac{h}{\rho r} \frac{\partial(h r)}{\partial r}+\frac{G m}{r}-\frac{v^{2}}{r}=0 \\
& \frac{\partial h}{\partial t}+u \frac{\partial h}{\partial r}+\frac{h}{r} \frac{\partial(u r)}{\partial r}=0 \\
& \frac{d}{d t}(v r)=0 \\
& \frac{\partial e}{\partial t}+u \frac{\partial e}{\partial r}-\frac{p}{\rho^{2}}\left[\frac{\partial \rho}{\partial t}+u \frac{\partial \rho}{\partial r}\right]+\frac{1}{\rho r} \frac{\partial(j r)}{\partial r}=0 \\
& \frac{\partial m}{\partial r}=2 \pi \rho r \\
& \frac{\partial j}{\partial r}=K j
\end{aligned}
$$

where $\mathrm{u}, \mathrm{p}, \rho, \mathrm{h}, \mathrm{m}, \mathrm{v}, \mathrm{j}$ and e are the radial component of velocity, pressure , density, magnetic field, mass per unit volume, azimuthal component of velocity, monochromatic radiation flux, energy per unit mass at a radial distance $r$ and time $t$ respectively. $\mathrm{G}$ represents the gravitational constant and $\mathrm{K}$ is the absorption coefficient.

$$
\text { Moreover, } \mathrm{e}=\mathrm{p} /\{\rho(\gamma-1)\}
$$

where $\gamma$ is the ratio of specific heats.

The radial velocity $\mathrm{u}_{0}$, azimuthal component of velocity $\mathrm{v}_{0}$, density distribution $\rho_{0}$, magnetic field $h_{0}$ in the undisturbed medium are taken as

$$
\mathrm{u}_{0}=0 ; \mathrm{v}_{0}=0 ; \rho_{0}=\rho^{*} \mathrm{R}^{\beta},(-2<\beta \leq 0) ; \mathrm{h}_{0}=\mathrm{h}^{*} \mathrm{R}^{-1}
$$

where $\rho^{*}, h^{*}, \beta$ are constants, $h_{0}$ is assumed to be directed tangentially to advancing shock front and $\mathrm{R}$ is the radius given by $(\mathrm{dR} / \mathrm{dt})^{2}=\mathrm{A}^{2} \mathrm{R}^{-\alpha}, \mathrm{A}$ and $\alpha$ are constants.

The boundary conditions at the shock are given by [Zel'dovich and Raizer [4]:

$$
\begin{array}{cc}
\mathrm{U}_{1}=2(\mathrm{dR} / \mathrm{dt}) /(\gamma+1), & \mathrm{v}_{1}=2(\mathrm{dR} / \mathrm{dt}) /(\gamma+1) \\
\rho_{1}=\left\{\rho_{0}(\gamma+1)\right\} /(\gamma-1), & \mathrm{p}_{1}=2 \rho_{0}(\mathrm{dR} / \mathrm{dt})^{2} /(\gamma+1), \\
\mathrm{e}_{1}=2(\mathrm{dR} / \mathrm{dt})^{2} /(\gamma+1)^{2}, & \mathrm{~h}_{1}=\mathrm{h}_{0}(\gamma+1) /(\gamma-1), \\
\mathrm{m}_{1}=\mathrm{m}_{0}=2 \pi \rho^{*} \mathrm{r}^{2+\beta} /(2+\beta) &
\end{array}
$$

where the suffixes ' 1 ' and ' 0 ' refer to the conditions just behind and ahead of the shock respectively. 
The Alf'ven Mach number and the usual Mach number are defined as $M_{A}{ }^{2}=\frac{\rho_{0} V^{2}}{h_{0}{ }^{2}}$ and $M^{2}=\frac{\rho_{0} V^{2}}{\left(2 p_{0}\right)} \quad$ respectively where $\mathrm{V}=\mathrm{dR} / \mathrm{dt}$ is the speed of the shock.

The absorption co-efficient $\mathrm{K}$ is considered as ( Nath [1] ):

$$
\mathrm{K}=\mathrm{K}_{0} \rho^{\mathrm{n}} \mathrm{p}^{\mathrm{m}} \mathrm{j}^{\mathrm{q}} \mathrm{r}^{\mathrm{s}} \mathrm{t}^{1}
$$

where the dimension of the constant $\mathrm{K}_{0}$ is given by

$\left[\mathrm{K}_{0}\right]=\mathrm{M}^{-\mathrm{n}-\mathrm{m}-\mathrm{q}} \mathrm{L}^{3 \mathrm{n}+\mathrm{m}-\mathrm{s}} \mathrm{T}^{2 \mathrm{~m}+3 \mathrm{q}-1}$

Moreover, the dimensionless constants $\mathrm{J}_{0}, \mathrm{p}_{0}, \rho_{0}$ are related as

$$
j_{0}=p_{0}^{3 / 2} \rho_{0}^{-1 / 2}
$$

Under the equilibrium condition, we have from (2)

$$
G=-\left[1 /\left(\gamma M^{2}\right)+1 /\left(2 M_{A}^{2}\right)\right](2+\beta)(1+\beta)(d R / d t)^{2} /\left(\pi \rho^{*} r^{\beta+2}\right)
$$

\section{SOLUTION OF THE PROBLEM}

Let us assume the solution of the fundamental equations (1)-(8) in the similarity form as $u=(d R / d t) U(x)$,

$v=(d R / d t) V(x)$,

$\rho=\rho^{0} F(x)$,

$p=\rho^{0}(d R / d t)^{2} P(x)$,

$m=m_{1} S(x)$,

$h=\rho_{0}^{1 / 2}(d R / d t) H(x)$,

$j=J_{0} J(x)$,

$e=(d R / d t)^{2} E(x)$,

$$
\text { where } X=r / R(t) \text {. }
$$

Substituting these in the fundamental equations (1) $-(8)$, we get a set of differential equations as given below:

$$
\begin{aligned}
& \beta F-X(d F / d X)+U(d F / d X)+F(d U / d X)+F U / X=0 \\
& (U-X)(d U / d X)-(\alpha U) / 2+[(d P / d X) / F]+H /(X F)[H+ \\
& X(d H / d X)]-\left\{1 /\left(\gamma M^{2}\right)+1 /\left(2 M_{A}^{2}\right)\right] 2(1+\beta)(S / X)-V^{2} / X=0 \\
& (\beta-\alpha) / 2+(U-X)(d H / d X) / H+U / X+(d U / d X)=0 \\
& U[V+X(d V / d X)]-X[(\alpha V / 2)+X(d V / d X)]=0 \\
& -[\alpha E+X(d E / d X)]+U(d E / d X)-\left(P / F^{2}\right)[\beta F+(U-X)(d F / d X)] \\
& +1 /\left(X F \gamma^{3 / 2} M^{3}\right)[J+X(d J / d X)]=0
\end{aligned}
$$




$$
\begin{aligned}
& \frac{d J}{d X}=\alpha_{1} X^{s} F^{n} P^{m} J^{q+1} \\
& \frac{d P}{d X}=(\gamma-1)[E(d F / d X)+F(d E / d X)] \\
& (29) \\
& \frac{d S}{d X}=[(2+\beta) F / X]-[(2+\beta) S / X] \\
& \alpha_{1}=K_{0} \rho_{0}^{n+m-1} j_{0}^{q+1}(d R / d t)^{2 m-2+s}
\end{aligned}
$$

The above set of differential equations (24)-(32), after some simplifications can be put in the following form:

$$
\begin{aligned}
& \frac{d U}{d X}=[(\alpha F U X(U-X)) / 2+\gamma P U+(\beta-\alpha) P X \\
& +(\gamma-1)\left(1 / \gamma^{3 / 2} M^{3}\right)\left(\alpha_{1} X^{s+1} F^{n} P^{m} J^{q+1}+J\right)+\left(X H^{2} / 2\right)(\beta-\alpha+2) \\
& \left.+\left(1 / \gamma M^{2}+1 / 2 M_{A}^{2}\right) 2(1+\beta) S(U-X) F+V^{2} F(U-X)\right] \\
& \div\left(F X(U-X)^{2}-\gamma P X-X H^{2}\right) \\
& \frac{d F}{d X}=-(F /(U-X))[(U / X)+(d U / d X)+\beta] \\
& \frac{d H}{d X}=-(H /(U-X))[(U / X)+(d U / d X)+(\beta-\alpha) / 2] \\
& \frac{d E}{d X}=-(E /(U-X))[(\gamma-1)((U / X)+(d U / d X)) \\
& \left.+((\gamma-1) / P X)\left(1 / \gamma^{3 / 2} M^{3}\right)\left(\alpha_{1} X^{s+1} F^{n} P^{m} J^{q+1}+J\right)-\alpha\right] \\
& \frac{d P}{d X}=(\gamma-1)[E(d F / d X)+F(d E / d X)] \\
& \frac{d S}{d X}=[(2+\beta) F / X]-[(2+\beta) S / X] \\
& \frac{d J}{d X}=\alpha_{1} X^{s} F^{n} P^{m} J^{q+1} \\
& +V /(U-x))[(U / X)-\alpha / 2] \\
& +(U)
\end{aligned}
$$


The boundary conditions (10) changes to-

$\mathrm{U}=2 /(\gamma+1), \mathrm{V}=2 /(\gamma+1), \mathrm{F}=1, \mathrm{P}=1, \mathrm{E}=1, \mathrm{~J}=1, \mathrm{H}=1$ and $\mathrm{S}=1$ for $\mathrm{X}=1$.

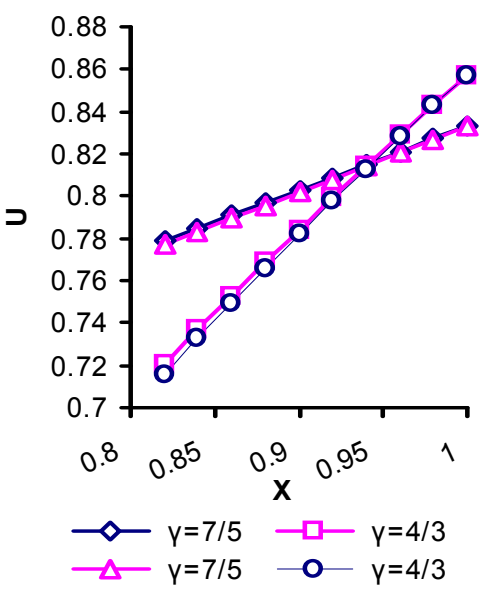

Fig 1. Variation of Radial velocity with Distance series $1 \& 2$ shows with Gravitation $(\gamma=7 / 5,4 / 3) \quad \&$ series $3 \& 4$ without Gravitation $(\gamma=7 / 5,4 / 3)$



Fig 3. Variation of Magnetic Field with Distance series $1 \& 2$ shows with Gravitation $(\gamma=7 / 5,4 / 3) \&$ series $3 \& 4$ without Gravitation $(\gamma=7 / 5,4 / 3)$

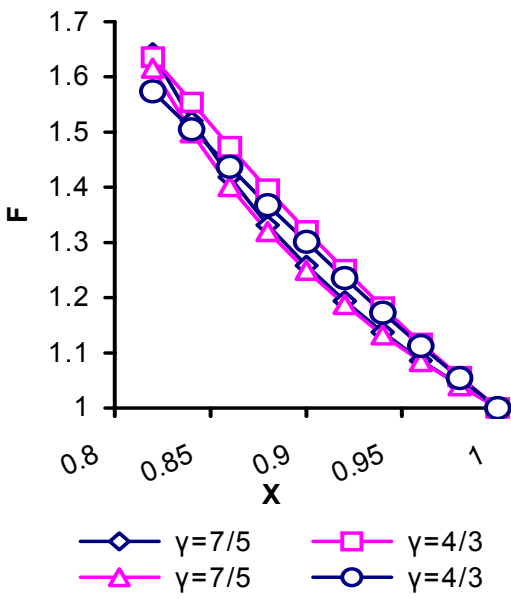

Fig 2. Variation of Density with Distance series1\&2 shows with Gravitation $(\gamma=7 / 5,4 / 3) \&$ series $3 \& 4$ without Gravitation $(\gamma=7 / 5,4 / 3)$

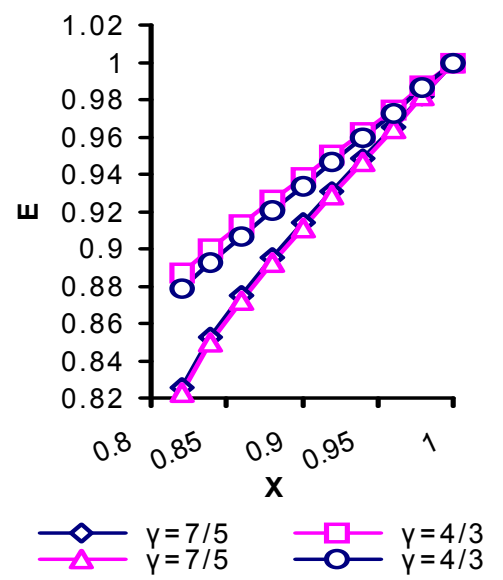

Fig 4. Variation of Energy with Distance series $1 \& 2$ shows with Gravitation $(\gamma=7 / 5,4 / 3) \&$ series $3 \& 4$ without Gravitation $(\gamma=7 / 5,4 / 3)$ 


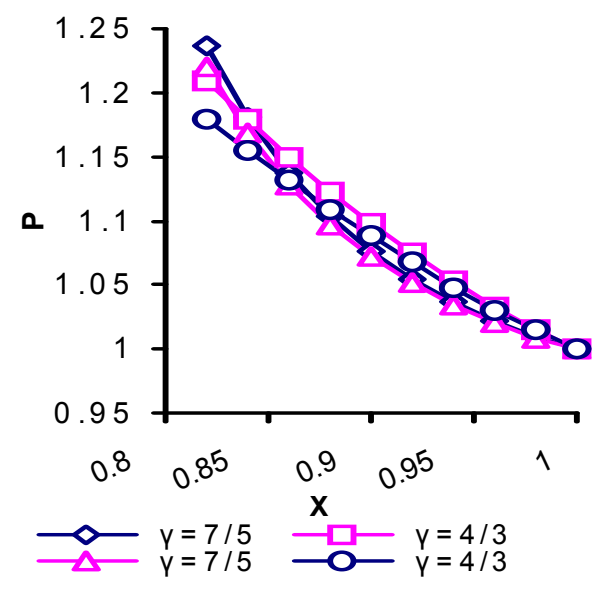

Fig 5. Variation of Pressure with Distance series $1 \& 2$ shows with Gravitation $(\gamma=7 / 5,4 / 3)$ \& series $3 \& 4$ without $\operatorname{Gravitation}(\gamma=7 / 5,4 / 3)$

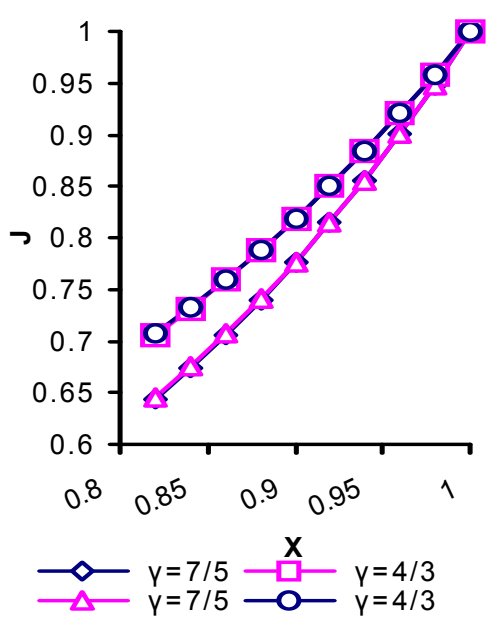

Fig 7. Variation of Radiation Flux with Distance series1\&2 shows with Gravitation $(\gamma=7 / 5,4 / 3) \&$ series $3 \& 4$ without Gravitation $(\gamma=7 / 5,4 / 3)$

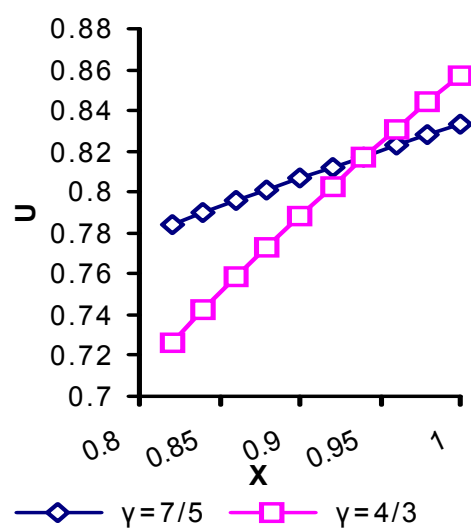

Fig 9. Variation of Radial velocity with Distance

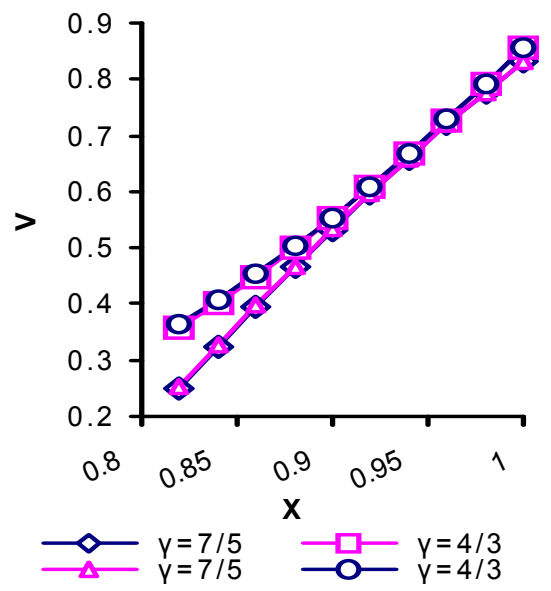

Fig 6. Variation of Rotational Velocity with Distance series $1 \& 2$ shows with Gravitation $(\gamma=7 / 5,4 / 3)$ \& series $3 \& 4$ without Gravitation $(\gamma=7 / 5,4 / 3)$

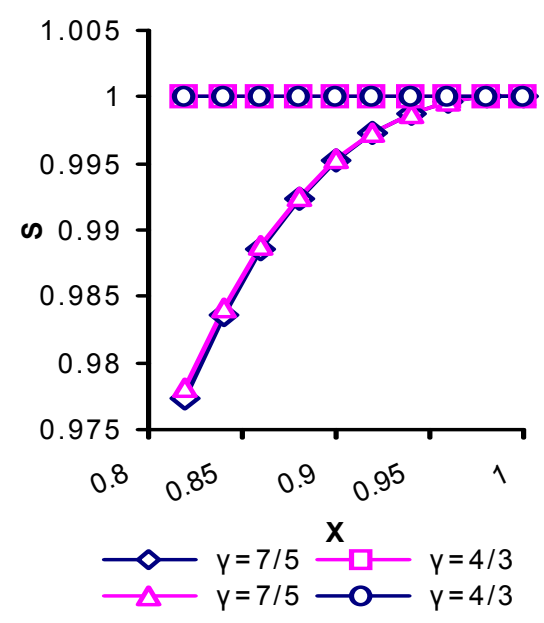

Fig 8. Variation of Mass with Distance series $1 \& 2$ shows with Gravitation $(\gamma=7 / 5,4 / 3) \&$ series $3 \& 4$ without $\operatorname{Gravitation}(\gamma=7 / 5,4 / 3)$

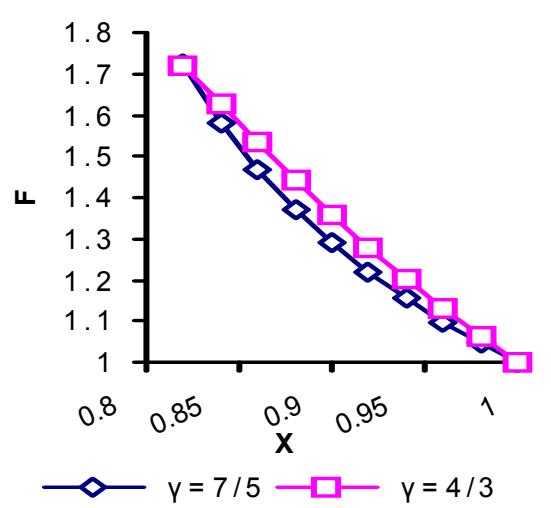

Fig 10. Variation of Density with Distance 

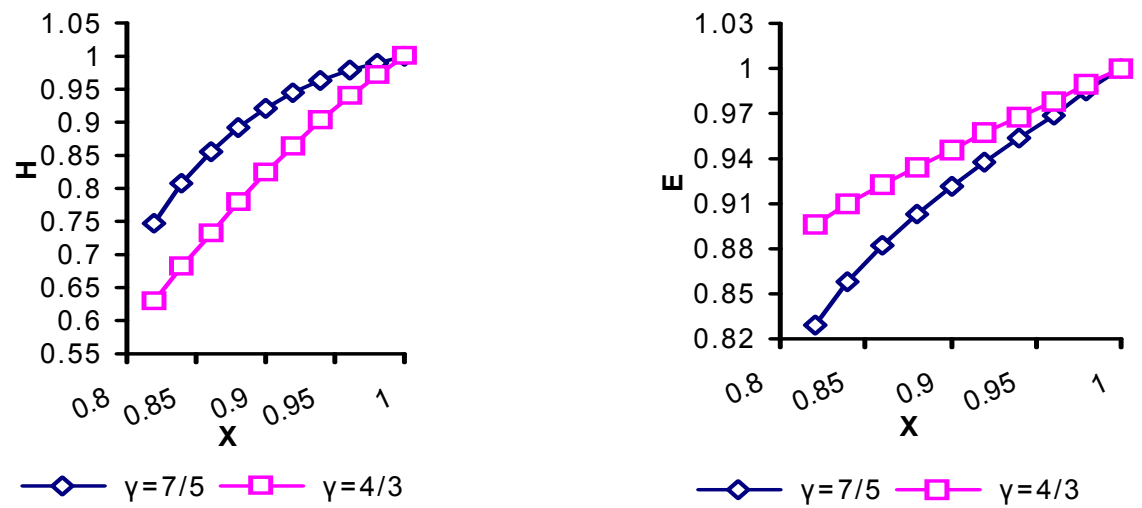

Fig 11. Variation of Magnetic Field with Distance

Fig 12. Variation of Energy with Distance
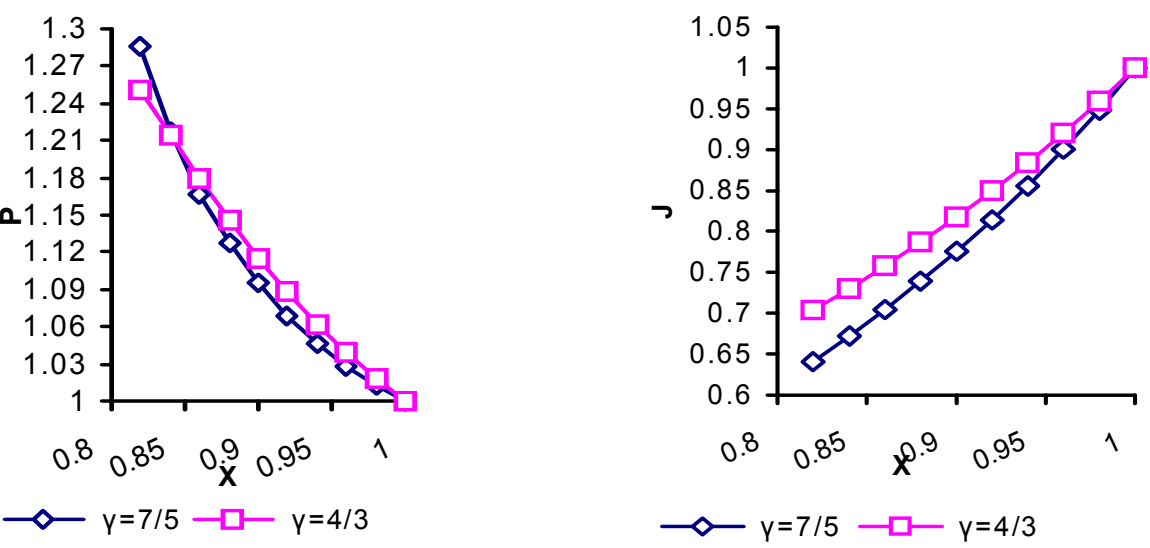

Fig 13. Variation of Pressure with Distance

Fig 14. Variation of Radiation Flux with Distance

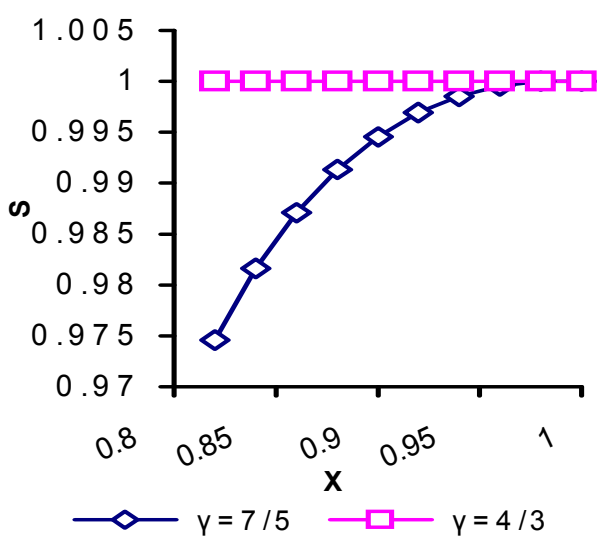

Fig 15. Variation of Mass with Distance 


\section{RESULT AND DISCUSSIONS}

The set of differential equations (33)- (40) have been integrated numerically with the help of boundary conditions (41) by the well known Runge -Kutta method $\alpha_{1}=2$ and 2.5, $\beta=-1.5$ and $-2, \gamma=4 / 3,7 / 5 ; M^{2}=5, M_{A}{ }^{2}=10, \alpha=2(2-\gamma) /(\gamma+1)$ and $n=-1 / 2, m=3 / 2$, $\mathrm{q}=0, \mathrm{~s}=1$. We have plotted the graphs showing the variations of various flow parameters with distance for different values of $\gamma, \beta$ and $\alpha_{1}$ in presence and absence of gravitational field and in presence and absence of rotation. This helped us to study the importance of gravitation and rotation respectively on flow parameters.

From Fig.1 to Fig.8, we observe that the radial component of velocity, magnetic field, energy, rotational velocity, radiation flux decrease as we go towards the center of the explosion, while density and pressure increase as we go towards the center of the shock. In fig.8, it is surprising to note that the mass decreases for the case of $\gamma=7 / 5$ for both presence and absence of gravitational field, it remains uniform for the case of $\gamma=4 / 3$. In absence of gravitational field decrease in the radial velocity, magnetic field and energy is more prominent. In the presence of gravitational field we see that decrease in rotational velocity, radiation flux is more prominent while the increase in the value of density and pressure is more prominent.

To draw a comparison between gravitational effects vis-à-vis rotational effect, we have also observed the variations of flow parameters in the absence of rotation in fig. 9 to fig. 15 and compared it with the earlier drawn graphs for the absence of gravitation. We find that radial velocity, magnetic field, energy decrease more in presence of rotational velocity while radiation flux decreases more rapidly in the absence of rotational velocity. As expected, in the absence of rotation, the value of pressure and density increase more as we go towards the center of the shock. However, the peculiar phenomenon to be observed is that the variation of mass remains uniform in case of $\gamma=4 / 3, \alpha_{1}=2$ and $\beta=-2$ irrespective of the presence or absence of the rotational effect. We further note that the gravitational effect is important for the propagation of shock waves in the present problem.

\section{REFERENCES}

1. Onkar Nath, A study of self-similar cylindrical MHD shock waves in monochromatic radiation, Astrophysics and Space Science, 155,163167,1989 .

2. Onkar Nath, Propagation of cylindrical shock waves in a rotating atmosphere under the action of monochromatic radiation, IL,NUOVO CIMENTO, 20D,N.12, 1845-1852,1998.

3. L. I. Sedov, Similarity and dimensional methods in mechanics, Academic Press, New York, 1959.

4. Ya. B. Zel'dovich and Yu. P. Raizer, Physics of Shock Waves and High Temperature Hydrodynamic Phenomena, Academic Press, New York, 1967. 